\title{
SPECTRAL PROPERTIES AND NEW INTERPOLATING SOLUTION OF ANDERSON MODEL
}

\author{
A.L. KuzEMSKY \\ Bogolubov Theorelical Laboratory, Joint Institute for Nuclear Research \\ 141980 Dubna, Moscow Region, Russia
}

\begin{abstract}
A new approach to many-body quasiparticle dynamics of the Anderson impurity model at finite temperatures is proposed in the framework of the equation-of-motion method. We find a new exact identity, which relates the one-particle and many-particle Green functions. Using this identity, it is shown that a new interpolating approximation for the one-particle Green function can be constructed. The approximation which simultaneously reproduces the weak-coupling limit and the strong coupling limit can be formulated self-consistently. This approach offers a new method for a systematic construction of the approximate interpolating dynamical solutions of strongly correlated electron models.
\end{abstract}

PACS numbers: $67.40 . \mathrm{Db}, 71.28 .+\mathrm{d}, 75.20 . \mathrm{IIr}$

The problem of an adequate description of strongly correlated electron systems has been studied intensively for the last decade, especially in the context of heavy fermions and high- $T_{\mathrm{c}}$ superconductivity [1]. Theoretical description of strongly correlated electron systems involves simplified model Hamiltonians. These includes as workable patterns single-impurity Anderson model (SIAM) and Hubbard model. Recently, a lot of effort has been made to gain a better insight into the dynamical properties of the Anderson/Iubbard model [1-16], especially in the context of many impurities case (e.g. [6]). This field is quite important for description of magnetic properties of anomalous rare-earth compounds [8]. The problem of an adequate and consistent description of dynamics of single-impurity and two-impurity Anderson models (SIAM and TIAM) and other models of correlated lattice electrons has not been fully analytically solved yet. The present paper is primarily devoted to the analysis of the relevant many-body dynamical solution of the SIAM and its correct functional structure. We wish to determine which solution actually arises from both the self-consistent many-body approach and intrinsic nature of the model itself. The IIamiltonian of SIAM has the standard form. We will consider single-particle GF of localized electrons. To improve 
the analysis of the problem we derive an exact new identity which can be written in a quite compact form

$$
\begin{aligned}
& \left\langle\left\langle f_{0 \sigma} \mid f_{0 \sigma}^{+}\right\rangle\right\rangle=g^{0}+g^{0} P g^{0}, \\
& g^{0}=\left[\omega-E_{0 \sigma}-S(\omega)\right]^{-1}, \\
& P=U\left\langle n_{0-\sigma}\right\rangle+U^{2}\left\langle\left\langle f_{0 \sigma} n_{0-\sigma} \mid f_{0 \sigma}^{+} n_{0-\sigma}\right\rangle\right\rangle_{\omega}, \\
& S(\omega)=\sum_{k} \frac{\left|V_{k}\right|^{2}}{\omega-\epsilon_{k}} .
\end{aligned}
$$

The benefit which Eq. (1) brings is that it does not include any approximation. IIere we shall use this identity as a basis for a new nonperturbative solution of SIAM in a wide range of temperatures and parameters of the model. We shall use the irreducible Green functions (IGF) method for the strongly correlated electronic systems $[10,11]$ too. In paper [2] the calculation of GF (1) has been considered in the limit of infinitely strong Coulomb correlation $U$. The functional structure of the Lacroix solution generalizes the well known "interpolative" solution [13] of SIAM. This solution gives correct results for $U=0$ and $V=0$. The important point about these two limits is that any approximate solution of SIAM should be consistent with them. Another attempt to give analytical solution of SIAM was made in papers [3, 4]. Unfortunately, the solutions given in Refs. [2, 3] do not reproduce correctly the $U$-perturbation expansion (cf. [5]) for the self-energy of GF (1). It is a highly non-trivial problem to find some way to incorporate this $U^{2}$ perturbation theory expansion in the functional structure of the interpolating dynamical solution of SIAM. A heuristic semi-empirical approach to constructing such a solution for SIAM has been presented in paper [15]. Our intention was to take into account this expansion in the self-consistent way. An attempt to do this was made in Ref. [16]. Let us consider the following equation of motion in the matrix form:

$$
\sum_{p} F(p, k) G_{\sigma}(p, \omega)=I+\sum_{p} V_{p} D(p, \omega),
$$

where $G$ is the initial $4 \times 4$ GF matrix and $D$ is a higher-order GF. Let us define the generalized mean field (GMF) GF according to

$$
\sum_{p} \tilde{F}(p, k) G_{\sigma}^{\mathrm{MF}}(p, \omega)=I,
$$

then we will be able to write down explicitly the Dyson equation and the exact expression for the self-energy $M$ in the matrix form. The explicit expressions for the matrix elements of our GMF solution (6) were presented in [9].

If we insert our GMF solution (6) into (3) we will get an essentially new dynamical solution of SIAM which reproduces the solutions for $V=0$ and $U=0$ and generalizes (even on the mean-field level) the solutions of papers $[2,3]$.

The identity (1) permits also a reformulation of the problem of derivation of the suitable interpolative solution of the SIAM, including the $U$-perturbation 
on the level of the higher-order GFs [16]. We start from the exact relation (1). The problem is to find the best possible fit for the higher-order GF in (3). The procedure requires to consider the equation of motion for it and then to treat them in an approximate way. This will give us a new approximative dynamical solution of SIAM in which the complex expansion in both $U$ and $V$ has been incorporated. The important observation is that this new solution satisfies both the above mentioned limits. Therefore it becomes possible to incorporate the $U$-perturbation expansion into our new solution for GF (2). To find an interpolating solution we require that

$$
\begin{aligned}
& \left\langle\left\langle f_{0 \sigma} f_{0-\sigma}^{+} c_{k-\sigma} \mid f_{0 \sigma}^{+} n_{0-\sigma}\right\rangle\right\rangle \approx \frac{1}{\left\langle n_{0 \sigma}\right\rangle\left\langle n_{0 \sigma} n_{0-\sigma}\right\rangle} \\
& \quad \times \int \mathrm{d} E_{1} \int \mathrm{d} E_{2} \int \mathrm{d} E_{3} \frac{\left[f\left(E_{1}\right)-f\left(E_{3}\right)\right] f\left(E_{2}\right)+f\left(E_{3}\right)\left[1-f\left(E_{1}\right)\right]}{\omega-E_{1}-E_{2}-E_{3}} \\
& \quad \times \operatorname{Im}\left\langle\left\langle f_{0 \sigma} \mid f_{0 \sigma}^{+} n_{0-\sigma}\right\rangle\right\rangle_{E_{1}} \operatorname{Im}\left\langle\left\langle c_{k \sigma} \mid n_{0 \sigma} f_{0-\sigma}^{+}\right\rangle\right\rangle_{E_{2}} \operatorname{Im}\left\langle\left\langle f_{0-\sigma} n_{0 \sigma} \mid f_{0-\sigma}^{+}\right\rangle\right\rangle_{E_{3}} \\
& \quad+\frac{\left\langle f_{0-\sigma}^{+} c_{k-\sigma} n_{0 \sigma}\right\rangle}{\left\langle n_{0 \sigma}\right\rangle}\left\langle\left\langle f_{0 \sigma} n_{0-\sigma} \mid f_{0 \sigma}^{+}\right\rangle\right\rangle,
\end{aligned}
$$

and analogously for the GF $\left\langle\left\langle c_{k-\sigma}^{+} f_{0 \sigma} f_{0-\sigma} \mid f_{0 \sigma}^{+} n_{0-\sigma}\right\rangle\right\rangle$.

Equation (7) gives a generalization of the $U^{2}$-perturbation-type expansion on the level of higher-order GFs. We have justified the use of approximation (7) in [16]. It was shown that the approximation (7) simultaneously reproduces the weak-coupling limit up to the second order in the interaction strength $U$ and the strong coupling limit up to the second order in the hybridization $V$ (and thus also fulfills the atomic limit) in a self-consistent way. We presented in this paper a consistent many-body approach to analytical dynamical solution of SIAM at finite temperatures and for a wide range of the model parameters values. The main results of our IGF study are the exact Dyson equation for the full $4 \times 4 \mathrm{GF}$ matrix and the new derivation of the GMF GF (6). We believe that our approach offers a new way for the systematic constructions of the approximative dynamical solutions of strongly correlated electron systems. The work in this direction is in progress.

\section{References}

[1] A.L. Kuzemsky, in: Superconductivity and Strongly Correlated Electron Systems, Eds. C. Noce, A. Romano, G. Scarpetta, World Scientific, Singapore 1994, p. 346.

[2] C. Lacroix, J. Phys. F, Met. Phys. 11, 2389 (1981).

[3] H.L. Neal, Phys. Rev. B 32, 5002 (1985).

[4] H.L. Neal, Phys. Rev. Lett. 66, 818 (1991).

[5] A.L. Kuzemsky, Phys. Lett. A 153, 466 (1991).

[6] A.L. Kuzemsky, J.C. Parlebas, H. Beck, Physica A 198, 606 (1993).

[7] A.L. Kuzemsky, in: Communications JINR, E17-96-23, Joint Institute of Nuclear Research, Dubna 1996; Int. J. Mod. Phys. B, to be published.

[8] A.L. Kuzemsky, JINR Rapid Commun. 3-03, 13 (1993). 
[9] A.L. Kuzemsky, Acta Phys. Pol. A 49, 169 (1976).

[10] A.L. Kuzemsky, Dokl. Akad. Nauk SSSR 309, 323 (1989).

[11] A.L. Kuzemsky, Nuovo Cimento B 109, 829 (1994).

[12] R. Taranko, A.L. Kuzemsky, Acta Phys. Pol. A 7G, 663 (1989).

[13] A. Hewson, Phys. Rev. 144, 420 (1966).

[14] A.L. Kuzemsky, in: Communications JINR, E17-95-205, Joint Institute of Nuclear Research, Dubna 1995.

[15] J. Ferrer, A. Martin-Rodero, F. Flores, Phys. Rev. B 36, 6149 (1987).

[16] G. Czycholl, A.L. Kuzemsky, S. Wermbter, in: Communicalions JINR, E17-95-297, Joint Institute of Nuclear Research, Dubna 1995; Europhys. Lett. 34, 133 (1996). 